\title{
Germanica
}

GERMANICA

31 | 2002

Le travail de réécriture dans la littérature de langue allemande au XXe siècle

\section{Franz Werfel : Jeremias. Höret die Stimme, roman de la protestation ou le courage de la confiance}

Franz Werfel: Jeremias. Höret die Stimme, Protest und Mut zum Vertrauen

\section{Bernard Bach}

\section{OpenEdition}

\section{Journals}

Édition électronique

URL : http://journals.openedition.org/germanica/2067

DOI : 10.4000/germanica.2067

ISSN : $2107-0784$

Éditeur

Université de Lille

Édition imprimée

Date de publication : 31 décembre 2002

Pagination : 23-38

ISBN : 9782913857100

ISSN : 0984-2632

Référence électronique

Bernard Bach, «Franz Werfel : Jeremias. Höret die Stimme, roman de la protestation ou le courage de la confiance », Germanica [En ligne], 31 | 2002, mis en ligne le 19 mars 2013, consulté le 06 octobre 2020. URL : http://journals.openedition.org/germanica/2067 ; DOI : https://doi.org/10.4000/germanica.2067

Ce document a été généré automatiquement le 6 octobre 2020.

(c) Tous droits réservés 


\section{Franz Werfel : Jeremias. Höret die Stimme, roman de la protestation ou le courage de la confiance}

Franz Werfel: Jeremias. Höret die Stimme, Protest und Mut zum Vertrauen

Bernard Bach

1 Le roman Jeremias. Höret die Stimme est paru en 1937 dans un contexte européen marqué à la fois par la montée d'un antisémitisme radical, qui ne tardera pas à déployer toute son horreur, et par l'impérialisme allemand dont la politique extérieure mise en œuvre de 1933 à 1936 allait donner à l'Allemagne hitlérienne l'initiative en Europe, ce qui ne pouvait qu'inquiéter et alarmer les observateurs lucides. Franz Werfel est un esprit cosmopolite, toute son œuvre est imprégnée de l'esprit du temps. On y a vu par ailleurs les fragments d'une grande confession ${ }^{1}$. Au moment où Werfel commence à écrire ce grand roman biblique, il traverse une crise de la création ${ }^{2}$ et une crise d'identité. D'origine juive, il avait manifesté depuis longtemps sa sympathie pour le christianisme. L'évolution du contexte politique des années trente mettait Werfel dans une situation pour le moins inconfortable et suscitait des interrogations angoissées. L'auteur de Jeremias n'ignorait pas la menace qui pesait sur ses coreligionnaires ni les réactions que pouvaient susciter ses sympathies pour le christianisme.

2 L'histoire de Jérémie est insérée dans un cadre narratif qui à la fois établit la relation avec le présent et donne une première indication sur le sens du choix de ce sujet: Clayton Jeeves, un jeune écrivain anglais $(9,21)^{3}$ traverse une profonde crise $(13,15,20$, 21, 22, 25-27). Depuis la mort de sa femme Leonora, il a sombré dans une sorte de dépression et de désespérance qui a tari en lui sa force créatrice (21). Mais le mal dont souffre Clayton Jeeves n'est pas seulement conjoncturel, il est plus profond, il est existentiel: C'est l'angoisse du vide et de l'absurde qui paralyse son affirmation créatrice $^{4}$. Cela provoque chez lui un sentiment de séparation de lui-même d'avec l'ensemble de la réalité, un sentiment d'isolement du soi comme individu ${ }^{5}$. Clayton Jeeves a comme l'impression d'avoir été attiré à Jérusalem contre son gré (25-26), en ce lieu mystérieux qu'il éprouve comme le centre du monde (Mittelpunkt der Welt 9, 10, 18). Ce 
qu'il est venu chercher ou redécouvrir en ce lieu, même s'il n'en pas immédiatement conscience, c'est ce centre spirituel qu'il est en train de perdre et qui donnait une réponse, symbolique, à la question de la signification de l'existence et sur lequel peut s'appuyer l'affirmation créatrice de soi. Le voyage à Jérusalem exprime symboliquement la quête de ce centre essentiel susceptible de guérir Clayton de son mal existentiel.

3 La question qui se pose dès lors est de savoir quelles forces de guérison recèle l'histoire de Jérémie pour Clayton. En d'autres termes, quelles réponses Clayton a-t-il puisé dans l'histoire de Jérémie pour surmonter sa crise existentielle? Clayton ne reprend pas intégralement l'histoire de Jérémie, il la relit d'une certaine façon, la recompose, actualise des potentialités, modifie la perspective première du texte source, bref, il la réécrit en fonction de ses préoccupations. C'est ce travail de réécriture que nous nous proposons d'examiner en vue de dégager la visée de la réécriture de l'histoire de Jérémie et de préciser la fonction de ce récit dans la crise que traverse Werfel dans le contexte historique, politique et social de la fin des années trente.

4 Le prophète Jérémie a vécu les quarante dernières années du royaume de Juda, il a exercé son métier de prophète dans une des périodes les plus mouvementées de l'histoire d'Israël. Franz Werfel reprend trois aspects essentiels du livre de Jérémie dont il développe et amplifie l'importance: la protestation contre le monde, la souffrance endurée en retour et l'affirmation du courage de la confiance qui s'appuie sur une expérience unique et personnelle de la transcendance.

\section{La protestation contre le monde}

5 Jérémie est l'homme du conflit, la protestation contre le monde se présente sous une triple forme: opposition entre le fils et sa famille, entre Jérémie et les dirigeants successifs du Royaume de Juda, entre le prophète et les religions égyptienne et babylonienne. Elle se situe au niveau psychologique, politique et religieux. Dans le livre de Jérémie la persécution du prophète par sa famille n'est mentionnée que brièvement dans les «Confessions » en Jr 11, 18-23 et Jr 12, 6. Franz Werfel reprend et amplifie le thème, notamment sous la forme du conflit entre le père et le fils. Hilkijah, vieux prêtre rempli d'amertume (81), refuse absolument la vocation prophétique de son fils (81) dont il veut faire un prêtre du temple (85). Il ne peut accepter la mise en cause d'un ordre, il est d'une lignée de prêtres, qui se conçoivent comme les gardiens scrupuleux de l'ordre du Temple ${ }^{6}$ et pour lesquels le prophète ne peut être qu'un fauteur de troubles et un agitateur politique ${ }^{7}$ sans véritable crédit. Jirmijah résiste au père et quitte la maison (86). Sa vocation prophétique implique une rupture avec son milieu familial. Seul le lien avec la mère est préservé. Mais l'image du père poursuit le prophète : lorsqu'au temple il ouvre pour la première fois le dossier d'accusation, il croit reconnaître dans la foule son père transformé en gardien du temple (124) et pointant un doigt accusateur sur lui. En réalité, le père meurt au même moment à la maison (124). Jirmijah sait qu'il est responsable de cette mort $^{8}$. Le prophète inspiré a tué le prêtre gardien de l'ordre, signe qui confirme que le prophète est un homme de rupture, dont la fonction première est celle de protestation contre un ordre perverti.

6 La vocation prophétique de Jirmijah le met également en opposition avec ses frères (127). La question de l'héritage ne cessera d'alimenter leur haine (129). Les malheurs que Jirmijah attire sur sa famille à cause de sa prédication provoqueront la rupture 
définitive avec ses frères, il n'y aura plus de réconciliation possible ${ }^{9}$. Mocheleth, l'épouse d'Obadjah, tentera même d'empoisonner Jirmijah (339). Lui et sa famille représentent deux mondes irréconciliables : la famille défend l'ordre d'un monde que le prophète remet fondamentalement en cause parce qu'il est devenu injuste et inhumain (325-326). L'élection divine a fait de Jirmijah un errant devenu étranger sur sa propre terre natale ${ }^{10}$.

7 L'engagement politique de Jirmijah le conduira à s'opposer à tous les souverains dont certains pourtant essaieront de s'attacher ses faveurs et le protégeront. Tout se passe comme si Jirmijah n'existait que par opposition. Pour réaliser le grand dessein de son règne, reconstruire le royaume de David $(145,146,148)$, le roi Josijah a besoin de la caution du prophète, il cherche ainsi à s'attacher les faveurs de Dieu $(159,168)$. Jirmijah, que le souverain a appelé à la cour (133), comprend très vite qu'il n'est qu'un instrument entre ses mains et que les puissants du royaume ne tiennent guère plus en estime le prophète que ne le faisait son père (150). L'enthousiasme guerrier du roi Josijah et des princes effraie le prophète (153). En dépit des avertissements répétés de Jirmijah $(163,166,167)$, Josijah engage la bataille de Meggido, il paiera de sa vie l'échec de cette folle ambition politique. Cet épisode ne figure pas dans le livre de Jérémie, mais

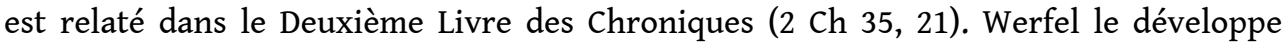
largement et insiste sur l'opposition irréductible entre le rêve de puissance de Josijah et les appels à la raison de Jirmijah (171).

8 Pour décrire les relations de Jirmijah avec le roi Jojakim, le successeur de Josijah, Werfel se réfère largement au Deuxième Livre des Rois et au Deuxième Livre des Chroniques, il reprend également quelques épisodes relatés dans le Livre de Jérémie : l'assassinat du prophète Urijah (Jr 26, 20-23), la destruction du rouleau (Jr 36), la poursuite de Jérémie et Baruch (Jr 36, 26). Jojakim, imposé sur le trône de Jérusalem par le vainqueur de Meggido, met en place un état policier s'appuyant sur les ressorts de la délation et de la propagande (226), et rêve à son tour de devenir le «nouveau David» (227). Mais ce roi cruel est également présenté comme un roi décadent ${ }^{11}$ devenu infidèle à son Dieu et à son peuple (296). Jirmijah formule contre le roi Jojakim un réquisitoire implacable (299-300) : il dénonce un roi rapace, tyrannique et violent (297), pressurant ses sujets pour enrichir son palais de constructions nouvelles ou décorations somptuaires et répandant à profusion le sang des innocents (226, 295-298). L'antagonisme profond qui oppose Jirmijah à ce monarque trouve son expression dans la reprise de l'oracle de Jérémie contre Yoyaqim (Jr 22, 13-19) : dans une intervention éclatante que Werfel situe en présence du roi, Jirmijah promet à Jojakim une sépulture d'âne (300). Mais le souverain ne tient aucun compte des mises en garde sévères de Jirmijah, il fait brûler le rouleau où Baruch a consigné les oracles du prophète (331) et poursuit sa politique de révolte contre Babylone. Comme Josijah, Jojakim cherche à s'attirer les faveurs de la divinité pour les besoins d'une politique à visée purement humaine (316). Dans les textes bibliques, les circonstances de la mort de Yoyaqim sont peu claires (2R 24, 6 et Jr $22,19 ; 36,30)$, Werfel présente la mort de Jojakim comme un suicide (362) indiquant ainsi que le monarque est la victime de sa propre folie.

Après la mort de Jojakim, Jirmijah entre très vite en conflit avec son successeur Zidkijah, dont il avait pourtant assuré l'éducation et en qui il avait mis toute sa confiance (377). Werfel reprend des éléments des Livres des Rois et des Chroniques ainsi que du livre de Jérémie. Il dramatise en particulier certains épisodes comme celui du joug $(J \mathrm{r} 27,1-15)$ : Ce geste symbolique de protestation contre la politique d'opposition à Babylone se 
heurte à l'incompréhension générale (415-416). Mais malgré son isolement (417), Jirmijah se montre inflexible face à un roi présenté comme faible et prisonnier de la coalition qu'il a initiée (430) : il ne cesse de lui rappeler le respect de l'année sabbatique (429) et d'exiger la soumission à Babylone $(461-462,463,502-503)$ pour sauver le royaume de Juda. Il prédit la chute de Juda $(456,486)$ et le sort tragique de Zidkijah (482). Mais tous ses efforts restent vains. Le roi et le prophète représentent deux mondes fonctionnant selon des logiques irréductibles et irréconciliables. Comme Josijah et Jojakim, Zidkijah est tenté d'utiliser son Dieu pour la réussite de son entreprise humaine $(441,443)$. L'aveuglement de Zidkijah conduit à la chute du Royaume et à sa propre perte. Werfel présente avec une grande intensité dramatique la fin tragique du souverain (522-525) mentionnée en Jr 39, 5-7.

Si Jirmijah s'est opposé avec autant de constance aux rois de Juda et n'a cessé de défendre son option pour Babylone, il ne fait pas de cette option un absolu ni de Nebukadnezar son nouveau Dieu. Face au roi de Babylone, il manifeste la même liberté de parole que face aux rois de Juda. S'appuyant sur Jr 25, 12 et 28, 2 ainsi que sur Jr 50 et 51, Werfel met en scène l'opposition de Jirmijah à Nebukadnezar: Après le châtiment de Zidkijah, le prophète a la folle audace de déclarer devant le maître du monde que sa victoire n'est en réalité qu'un échec ${ }^{12}$, reprenant ainsi la prophétie qu'il avait faite autrefois devant ce même roi quand il était son invité (351).

11 Sur le plan religieux, Jirmijah affiche une triple opposition : opposition aux pratiques religieuses de son peuple, opposition à la religion égyptienne et opposition à la religion de Babylone. Jirmijah dénonce inlassablement les cultes païens qui perdurent en dépit de la réforme de Josijah $(67,108,109)$. Une de ses premières interventions publiques sera contre la prêtresse Frustra et le culte d'Aschera (109-110). Tout au long de sa prédication le prophète fustige le non respect de la loi sabbatique (voir Dt 15, 12-15), selon laquelle la liberté doit être rendue à tous les Hébreux dont la misère et le besoin ont fait des esclaves de leurs frères. Ce thème traverse tout le roman $(112,325,334$, 429), Jirmijah a fait du respect de la loi sabbatique le combat de sa vie, ce qui le conduit à s'opposer sans cesse à son peuple qui, pour des raisons économiques, ne respecte pas cette loi. Par ailleurs Jirmijah n'a pas de mots assez forts contre les faux prophètes qui orientent leur prédication en fonction des rapports de force du moment et de leurs intérêts personnels et chantent les louanges du souverain en place (285-287). Enfin, le prophète s'élève avec véhémence contre toute instrumentalisation du temple, des sacrifices et de Dieu lui-même ${ }^{13}$.

Le prophète, en proie au doute, est plusieurs fois tenté d'abandonner sa mission. Werfel consacre plusieurs chapitres au séjour qu'il fait faire à Jirmijah en Égypte, séjour dont les textes bibliques ne disent rien. L'auteur y montre un prophète pas insensible aux charmes de la religion égyptienne (187-188). Jirmijah y entame notamment un voyage initiatique à travers le royaume des morts (Der Gang durch die Amenti 237-256) au cours duquel il découvre l'inconsistance et la vacuité de la croyance égyptienne en la survie de l'âme (253-254). Il se produit comme un retournement de situation: Jirmijah a soudain la conviction qu'Adonai, son dieu, est le vrai et unique Dieu et qu'il existe un "salut plus élevé » et une "félicité plus digne » que celle des "Amenti» (254). Cela conduit Jirmijah à quitter l'Égypte et sa religion et à revenir à Jérusalem pour se remettre au service de son Dieu. renouveau, le prophète juif est l'invité d'honneur (392) de Mardukh-Nebukadnezar, il 
gravit la tour avec le roi et l'accompagne dans une sorte de voyage initiatique à travers les cieux (394-408) où il contemple avec ravissement le bel ordonnancement du monde (400). Jirmijah est prêt d'oublier son Dieu, Jérusalem et son peuple, quand la formule magique de Mardukh le tire brusquement de son ravissement céleste. Le prophète abusé comprend que la vision de l'harmonie stellaire est aussi illusoire que celle du royaume des morts en Égypte ${ }^{14}$. Rejetant alors la religion de Babylone, Jirmijah proclame devant Mardukh et l'univers céleste qu'Adonai est le Dieu éternel et unique ${ }^{15}$.

Par ses paroles et ses actes publics, Jirmijah démontre que le prophète est un homme de rupture, chargé de rappeler aux souverains leurs devoirs envers le Dieu unique de l'Ancien Testament et le peuple juif et de les mettre en garde contre les mortelles ambitions humaines, oublieuses de la source qui fait vivre le peuple. Le prophète apparaît d'autre part comme l'homme de la fidélité à une puissance transcendante dont il tient sa légitimité et au nom de laquelle il parle. Intervenant dans une des périodes les plus mouvementées et les plus incertaines de l'histoire du peuple juif, Jirmijah ne cesse d'affirmer avec force cette identité juive dont la composante essentielle est la fidélité à Yahvé et pour la défense de laquelle le prophète est prêt à mettre sa vie en jeu, car il sait que c'est de cette fidélité à Adonai que dépend l'avenir du peuple juif.

\section{Le chemin de croix de Jirmijah}

À propos du Livre de Jérémie on a souvent souligné que le prophète avait imposé à la tradition une toute nouvelle figure messianique, celle du serviteur souffrant ${ }^{16}$, que l'on retrouvera dans les chants du Serviteur d'Isaïe (Is 42, 1-9; 49, 1-7 ; 50, 4-11; 52, 13-53) et qu'il apparaissait en quelque sorte comme le précurseur du Messie. La figure de Jérémie ressemble étonnamment à celle du Christ lui-même. Werfel a repris et accentué cette figure du serviteur souffrant qu'il a doté d'une capacité de compassion infinie et de dépouillement de soi total, faisant ainsi de Jirmijah une authentique figure christique.

On notera que Jirmijah, à l'image de Jésus (Mc 1, 12-13; Mt 4, 1-11; Lc 4, 1-13), se retire au désert pour y jeûner avant d'entamer sa carrière de prophète. Cet épisode est d'autant plus significatif qu'il est inventé par Werfel. Toute la vie du prophète, qui se dévide sur 33 chapitres, un chiffre symbolique, ne sera qu'un chemin de passion. La vision de ce que sera la vie et le destin du prophète provoque cris et lamentations chez la voyante $\mathrm{Hulda}^{17}$. Au cours de sa prédication, Jirmijah est arrêté pas moins de cinq fois $(271,286,340,473,488)$, traduit devant le tribunal $(288,294$, 475), flagellé $(275,476)$, jeté en prison $(476,490)$, emmené en exil $(196,342,377,540)$. Le prophète affronte la haine de la foule ${ }^{18}$, il doit supporter le mépris et les railleries de ses compatriotes (276), il passe même à deux doigts de la mort quand, dans sa colère, Ismael lui lance un lourd chandelier à la tête (465), le défigurant à jamais (466-467). Certaines similitudes entre les traitements infligés à Jirmijah et à Jésus sont étonnantes. À certains égards, le lecteur peut avoir l'impression que la passion de Jésus s'est imposée en quelque sorte à Werfel comme modèle pour décrire celle de Jirmijah. Le roi Zidkijah a de l'estime pour Jirmijah, il sait que c'est un homme habité par Dieu, mais sous la pression de son entourage, qui hait le prophète, il cède et le livre à Ismael décidé à faire périr ce prophète de malheur ${ }^{19}$. Cette attitude de lâcheté est à rapprocher de celle de Ponce Pilate qui sous la pression des autorités juives livre Jésus à ses bourreaux (Jn 18,31; 19,6). Dans les deux cas, les soldats expriment leur haine envers Jirmijah ${ }^{20}$ et Jésus (Mc 
$14,65 ; 15,19)$ par des gestes d'humiliation absolument identiques ${ }^{21}$ que Jirmijah, comme Jésus, supporte en silence (489). Comme Jésus, Jirmijah atteint un sommet dans cette capacité de souffrance absolument unique dans l'histoire ${ }^{22}$. L'idée de la souffrance rédemptrice, du sacrifice pour la multitude, associée à la mort de Jésus (Mt 20,28;26, 28), est bien présente dans l'esprit de Jirmijah (420).

Un autre trait, fortement accentué par Werfel, rapproche Jirmijah de la figure du Christ: sa capacité de compassion. Jirmijah est inflexible devant les souverains, il ne cesse de s'opposer à eux, de leur décrire les conséquences désastreuses de leurs ambitions de puissance, mais lorsqu'ils sont vaincus, humiliés, châtiés, exilés, Jirmijah les accompagne, les réconforte, les console. Le prophète accueille dans son giron la tête de Josijah blessé à mort ${ }^{23}$, il l'accompagne "trois jours et trois nuits» (178) dans son agonie. Traîné devant le roi Jojakim pour atteinte à l'ordre public, Jirmijah est pris de compassion pour l'enfant sans vie du souverain et il le réanime (301). Après la destitution de Konjah, dont le règne n'a duré que quelques mois, Jirmijah ne peut se résigner à abandonner le pauvre souverain emmené en exil (376), il l'accompagne (377) et se fait son serviteur discret et muet malgré la haine implacable que lui voue le jeune monarque $^{24}$. On voit même le prophète verser des larmes sur le sort du malheureux (383) et intercéder pour lui auprès du puissant Nebukadnezar (404). La compassion de Jirmijah atteint un sommet face à Zidkijah qu'il a pourtant durement combattu, par qui il a été jeté en prison, puis livré à ceux qui voulaient le faire périr. Au moment du châtiment, le prophète est au pied de l'échafaud (521), dans son angoisse, le souverain déchu cherche son regard (523), après le terrible châtiment, Jirmijah monte sur l'échafaud, accueille la tête du souverain dans son giron ${ }^{25}$, lui prodigue les premiers soins et trouve les paroles de réconfort et de consolation (525). Quand au moment du siège de Jérusalem les oracles de destruction et de châtiment proférés par les prophètes implacables sèment la panique et le désespoir dans le peuple, Jirmijah est pris de compassion $^{26}$, il élève sa voix forte et profonde pour proclamer un oracle de salut et réconforter le peuple (358). Enfin, pour réconforter l'Ethiopien Ebedmelech, Jirmijah lui annonce qu'en vertu de sa fidélité lui et sa descendance font désormais partie du peuple de Dieu (535). Cette idée du Dieu universel, que Jirmijah défend par ailleurs devant le prince de Libna ${ }^{27}$ est bien celle du Nouveau Testament. Par sa compassion infinie pour les hommes et les femmes qui souffrent et désespèrent, Jirmijah donne du Dieu d'Israël cette image de Père miséricordieux que proclame Jésus dans le Nouveau Testament.

Enfin un troisième trait prêté à Jirmijah par Werfel, et qui force un peu le modèle biblique, donne une connotation fortement christique au prophète du roman: la capacité de total dépouillement de soi, la "kénose ». Dès le début de son ministère, Jirmijah comprend que pour entendre la voix d'Adonai il doit se mettre totalement en situation d'écoute, renoncer complètement à soi-même, se vider de soi-même ${ }^{28}$. Jirmijah apprend, en somme, à être pauvre, c'est-à-dire à vivre en dépendance radicale vis-à-vis de son Dieu. Sa prédication et son action seront toujours conformes à la volonté d' Adonai, l'ancrage en Dieu sera son seul repère, l'incompréhension de ses auditeurs n'y changera rien. Dans la lettre que Jirmijah adresse aux exilés, l'abaissement de soi prend une dimension nouvelle: le prophète invite non seulement ses compatriotes à s'installer et à vivre en paix à Babylone, mais encore à prier pour les ennemis (408). Certes, Werfel ne fait ici que suivre textuellement le passage de Jr 29, 7, il n'empêche que la tendance globale à "christianiser " Jirmijah est confirmée par quelques autres faits et gestes de Jirmijah, parfois inventés de toutes pièces par Werfel : le prophète voit 
dans la défiguration infligée par Ismael un effacement de son identité et une naissance nouvelle ${ }^{29}$. N'est-ce pas le signe que de l'abaissement du prophète surgit l'homme nouveau, qu'à travers sa défiguration est scellée en quelque sorte une alliance nouvelle entre Dieu et l'humanité dont le modèle de réalisation est Jésus? Werfel reprend par ailleurs cet épisode de Jr 38,5 où le prophète est jeté dans une citerne, mais il y ajoute une interprétation du sens de cette situation, révélatrice de la coloration christique de son personnage: Jirmijah comprend soudain ce qu'est l'abaissement de Dieu, il a l'intuition de ce qu'est l'incarnation ${ }^{30}$. Avant la rupture définitive entre Zidkijah et Jirmijah, le prophète lance ces paroles au souverain «Ne comprends-tu pas que ce sacrifice est ta seule force? » (504), ces paroles définissent parfaitement la «kénose » que réalise le prophète lui-même et qui atteint son sommet tout à la fin du roman : Jirmijah suit sans résistance le «reste» qui fuit en Egypte, le pays de l'esclavage, il accepte son effacement de l'histoire du peuple juif comme l'accomplissement de la volonté de son Dieu ${ }^{31}$. Mais avant de disparaître définitivement, Jirmijah apprend, par l'intermédiaire d'un débris des Tables de la Loi, le sens de ce renoncement total à soimême : par le sacrifice suprême librement consenti la mort sera vaincue et le peuple vivra éternellement ${ }^{32}$. On ne peut s'empêcher de voir dans cette interprétation finale de l'effacement de Jirmijah, absente du livre biblique, poindre l'idée chrétienne du sacrifice de l'Agneau pascal vainqueur de la mort et des forces du mal.

Il y a en Jirmijah à la fois une volonté inflexible d'affirmation de soi, un courage d'être soi-même en dépit de toutes les résistances et une capacité infinie à participer à la souffrance de celui qui est perdu, un courage d'être en participant qui va jusqu'au dépouillement total de soi. Ces traits donnent une coloration fortement christique au Jérémie de l'Ancien Testament réécrit par Werfel. Il ne fait pas de doute que Werfel s'inscrit dans la tradition exégétique chrétienne qui a vu très tôt en Jérémie une préfiguration du Christ. Force est de constater que Werfel assume le double héritage judéo-chrétien. Mais le problème n'est pas de déterminer quelle part de son identité Werfel trahirait avec ce roman, ce qu'il importe de comprendre c'est l'expérience fondatrice qui fait de Jirmijah cet homme de protestation, qui face à tous les pouvoirs manifeste une liberté irréductible, et le sens que revêt l'histoire du prophète pour Werfel lui-même et pour ses contemporains.

\section{L'affirmation du courage de la confiance}

Au fondement de la vie et de l'action du prophète Jirmijah il y a une expérience de la transcendance faite dans une rencontre unique et personnelle qui commence par un appel singulier, une élection. Un long combat s'engage alors entre le prophète et Dieu, peu à peu le prophète apprend à se soumettre à cette volonté transcendante qu'il ne comprend pas toujours. Sur ce point Werfel reprend les données du Livre de Jérémie, notamment le chapitre un (Jr 1, 4-19), mais comme à son habitude il les dramatise et les enrichit d'éléments puisés dans son imagination: dès sa première lecture publique au temple, le jeune Jirmijah est surpris par sa propre voix qui semble doublée par une autre voix (55), de même dans ses oracles, il aura parfois l'impression qu'une autre voix que la sienne parle à travers lui (120). Le roi Josijah comme les auditeurs remarquent d'emblée que la voix du jeune Jirmijah est habitée. Werfel présente Jirmijah comme un jeune homme à la psychologie fragile, dont les nuits sont hantées par des appels indistincts (68) qui le font parfois fuir dans la nature. L'appel de Dieu s'adresse au jeune 
homme sous la forme d'une voix d'homme à la fois profonde, nette et douce (71). Jirmijah répond à la manière de Samuel (1 S 3, 9-10): «Parle, Seigneur, ton serviteur écoute » (72). Werfel insiste sur la valeur fondatrice de cette première rencontre avec la transcendance, que Jirmijah vit comme une mort et une renaissance, l'appelé a clairement conscience qu'il est devenu un autre homme (75).

21 Mais la mission confiée au prophète n'a pas la clarté de l'évidence, Jirmijah, en répondant à l'appel, s'engage sur un chemin obscur et tortueux. La rencontre avec la transcendance revêt la forme d'un dur combat. Jirmijah est la proie du doute, il fait l'expérience d'un Dieu insaisissable, lointain, obscur, indéchiffrable (139), il consulte la voyante Hulda (97), le prophète Urijah doit le rassurer au sujet de sa vocation (96). Les rudes épreuves ébranlent plus d'une fois le prophète, il succombe à la tentation du renoncement. Après la mort incompréhensible de Josijah, sa confiance en la fidélité de Dieu vacille ${ }^{33}$. Le départ en Egypte apparaît alors, sous couvert de fidélité à la famille royale, comme un abandon de sa mission et une fuite devant Dieu (186). Sa décision de se marier en Égypte (215) ne fait que confirmer sa stratégie de démission. La mort de Zenua poussera même Jirmijah à se révolter contre Adonai ${ }^{34}$, et à maudire le jour de sa naissance (280). Mais ce Dieu insaisissable n'en continue pas moins à se manifester à Jirmijah en dépit de sa résistance (192). Le prophète découvre que son Dieu est un «feu brûlant » qui le consume de l'intérieur ${ }^{35}$.

De ce combat, c'est Dieu qui sort vainqueur. Après le meurtre de sa mère et son arrestation sur dénonciation, au moment où Jirmijah touche le fond de l'abîme, il tombe dans une prostration profonde (343), il est un esprit brisé qui n'a plus la force de se révolter, il reconnaît alors que Dieu est le plus fort ${ }^{36}$. Enfin, lorsque le royaume de Juda est détruit, le Temple de Jérusalem incendié et le Saint des Saints profané, Jirmijah, dans une ultime confrontation dramatique, obéit encore, le cœur brisé, à Celui qui lui demande d'accompagner le « reste » (529). Pour retracer cette expérience difficile de la rencontre avec la transcendance, Werfel s'est inspiré en grande partie de ce que l'on a appelé les «Confessions » de Jérémie (Jr 12, 1-5; 15, 10-21; 17, 14-18; 18, 19-23;20, 7-18). Il montre ainsi que le prophète n'est pas un surhomme, en décrivant avec force la détresse de cet homme qui ploie sous la charge que Dieu lui a confiée, il donne une dimension profondément humaine à Jirmijah, dont la grandeur réside finalement dans l'acceptation libre de son douloureux destin.

Dans ce combat singulier entre le prophète et Adonai, le vainqueur n'écrase pas le vaincu, mais le relève, lui donne la force d'exister, de s'affirmer dans le tourbillon du monde. Face au monde des puissants, Jirmijah a bien conscience que le prophète qu'il est ne représente rien (150). Il se sent trop faible pour la mission qu'Adonai lui confie (86), il manque de confiance en soi (89), il est torturé par l'angoisse (96), face à ses juges l'angoisse de la mort le fait chanceler ${ }^{37}$.

Comment ce prophète au cœur trop tendre et au tempérament trop sensible (556) peutil résister, seul $(417,453)$ contre tous ? D'où cet homme faible tient-il sa force? Ce qui est frappant, c'est que Jirmijah ne s'engage jamais sans avoir reçu de signe d'Adonai (345), avant de prendre une décision, d'oser une parole publique, il attend ce que Werfel appelle "eine Raunung $»^{38}$. Face aux puissants qui le pressent de leur donner l'oracle qu'ils attendent, face à la pression des grands et petits intérêts humains, face à ses juges, dans le vacarme des armes, Jirmijah patiente et reste à l'écoute de ce seul murmure sur lequel il fait reposer toute sa confiance. Et l'improbable finit par se produire, le prophète fait face, dans la tourmente il tient bon. En somme, c'est en 
renonçant à fonder sa confiance en soi-même et en s'appuyant sur Adonai seul et uniquement sur Adonai que Jirmijah parvient à acquérir la confiance dans sa propre existence, le courage de s'affirmer lui-même, en dépit de toutes les négativités dont il fait l'expérience, en dépit de l'angoisse qui le domine, en dépit de la solitude radicale à laquelle il est exposé dans le monde. Le courage d'être de Jirmijah s'enracine dans une puissance d'être qui est plus grande que la puissance du soi propre et que celle de son monde. Jirmijah se laisse saisir par la puissance de l'être lui-même et vit de celle-ci ${ }^{39}$. Ce courage de la confiance permet à Jirmijah d'intégrer une triple angoisse : l'angoisse de la culpabilité et de la damnation ${ }^{40}$, l'angoisse du vide et de l'absurde ${ }^{41}$ et l'angoisse du destin et de la mort $(270,489)^{42}$. Cela lui donne la force d'espérer même dans les situations sans issue. Alors que Jérusalem est prise en tenaille par les troupes de Babylone (445) et qu'une défaite imminente est certaine, Jirmijah provoque l'incompréhension générale en achetant un champ au prix fort (486-487). Par cette action, il manifeste qu'en dépit du caractère irrémédiable de la situation présente, sa participation à la puissance de l'être qui transcende tout ce qui est, lui donne la force d'affirmer un avenir possible.

Jirmijah apparaît comme le prophète incompris par son temps, qui n'a cessé de prédire la ruine du royaume de Juda et a finalement assisté, impuissant, aux défaites successives de trois souverains (Josijah, Jojakim et Zidkijah) et à la catastrophe finale, la destruction de Jérusalem et du Temple et la dispersion de son peuple. Le prophète attribue la cause fondamentale de ce désastre au refus des souverains de fonder l'affirmation de soi sur la participation à la puissance de l'être lui-même qui, en conjurant l'angoisse destructrice inhérente à la liberté, ouvre le véritable accès à un monde humain et fraternel. En se détournant d'Adonai, les rois successifs sont devenus les victimes de leurs ambitions démesurées, ils ont cherché à s'ériger eux-mêmes en absolu, entrant ainsi dans une logique de la concurrence impitoyable, destructrice pour eux-mêmes et pour le peuple tout entier ${ }^{43}$. C'est cette spirale infernale de la soif de puissance qu'illustrent les échecs successifs des souverains auxquels le prophète, par sa prédication et sa vie, rappelle inlassablement que sans l'acceptation d'un fondement absolu de l'existence humaine, les relations entre les hommes ne peuvent que dégénérer en luttes fratricides aux niveaux personnel et collectif.

En réécrivant la vie de Jérémie, Franz Werfel trace un portrait authentique du prophète de l'Ancien Testament, en cela il reste fidèle à la tradition biblique. On reconnaît bien en Jirmijah les principales caractéristiques du prophétisme biblique : en premier lieu, Jirmijah devient prophète non par décision personnelle, mais par obéissance à une volonté contraignante de nature transcendante. Jirmijah est tenté plusieurs fois d'abandonner sa mission, mais il finit toujours par la reprendre, vaincu par Adonai. En deuxième lieu, la vocation de Jirmijah est purement personnelle. Lui, qui est issu d'une lignée sacerdotale et dont le père rêve de faire un prêtre, entre ainsi en opposition avec sa famille et l'institution sacerdotale du Temple. Jirmijah refuse la sollicitation externe, qui est celle de son père, pour se soumettre à une voix intérieure, au murmure d'Adonai. La troisième caractéristique est que le prophète vit une forme d'aliénation, en ce sens qu'il se sent devenir un autre, comme s'il était possédé par Adonai dont il a une expérience vécue. Enfin le message de Jirmijah s'adresse à une communauté déterminée et à ses dirigeants ce qui entraîne des tensions avec l'ordre politique. Le prophète 
inquiète les autorités politiques et religieuses, il dérange les habitudes, conteste les situations d'injustice.

Jirmijah est au service d'une idée qu'il propage à ses risques et périls, indépendamment des institutions. Sa seule sécurité réside dans la force de sa parole, par laquelle il essaie de peser sur les événements sociaux et politiques. Surgissant dans une situation de crise politique, sociale et religieuse, il indique une voie à suivre, qui le met en permanence à contre-courant de l'histoire. Il apparaît ainsi comme celui qui reste souverainement libre à l'égard de l'attrait du pouvoir, du poids des habitudes, de la pression de l'opinion publique pour n'obéir qu'à son Dieu et mettre inlassablement son peuple devant la présence de ce Dieu.

En faisant sienne la tradition biblique, Werfel assume pleinement son identité juive. Mais le romancier réécrit également l'histoire de Jérémie d'une manière très personnelle, il donne à Jirmijah une connotation fortement christique. On reconnaît là l'influence de la problématique personnelle de l'auteur attiré par le christianisme. Werfel assume en somme le double héritage de la tradition judéo-chrétienne.

Mais l'essentiel est que l'histoire de Jérémie apporte d'une part une réponse à la crise existentielle que traverse Werfel et d'autre part un message clair aux hommes de son temps qui sont confrontés à la mortelle ambition d'un dictateur qui les entraînera dans la catastrophe. L'expérience «nouvelle et merveilleuse » (p. 553) vécue sur la plateforme du Temple par l'écrivain Clayton Jeeves se révèle être un " chemin de guérison ${ }^{44}$, qui a fortifié en lui son propre soi ${ }^{45}$ et lui a fait retrouver ses forces créatrices (p. 556). La voix du prophète biblique a traversé le temps ${ }^{46}$ pour répondre aux interrogations d'un homme du temps présent. On comprend dès lors le lien entre le cadre narratif et l'histoire de Jirmijah, le lien entre l'histoire passée et l'histoire présente : l'histoire passée du prophète apporte une réponse au mal existentiel dont souffre l'écrivain Clayton Jeeves, et à travers lui Werfel: Jirmijah lui montre que dans une époque bouleversée, menacée par la perte du sens et la destruction, l'ancrage dans l'absolu fait retrouver à l'homme ce centre essentiel qui non seulement le sauve du désespoir et lui permet de résister à la désintégration du moi, mais remet en branle l'affirmation créatrice et lui donne le courage de s'affirmer contre les forces de destruction. En même temps, le message de Jirmijah dépasse le cadre de l'individu et s'adresse à toute une époque : L'option fondamentale de Jirmijah est incompatible avec toutes formes de totalitarisme. Par sa protestation constante, Jirmijah démontre qu'aucun arsenal répressif, qu'aucun pouvoir totalitaire ne saurait réussir à faire taire celui qui n'écoute que la voix de sa conscience. C'est un appel indirect, mais clair, à entrer en résistance contre le nazisme. Le chemin douloureux de Jirmijah éclaire le temps de l'écriture. Son échec sonne comme une mise en garde à toute une époque, qui a proclamé la mort de Dieu et s'est laissée emporter dans le tourbillon des nationalismes, il a valeur prémonitoire. Mais l'effacement de soi auquel consent Jirmijah témoigne aussi de cette espérance millénaire dont est porteur le peuple juif. En dépit de l'avenir sombre qui se profile à l'horizon à la fin des années trente, Werfel ne veut pas désespérer. L'histoire, hélas, montrera que Werfel, pas plus que Jirmijah, n'aura réussi à inverser le cours des événements. 


\section{NOTES}

1. Cf. Anneliese Kuchinke-Bach : Franz Werfel, in : Deutsche Dichter. Vom Beginn bis zur Mitte des 20. Jahrhunderts. Bd 7. Stuttgart : Reclam, 1989, p. 380.

2. Cf. Peter Stephan Jungk : Franz Werfel. Eine Lebensgeschichte. Frankfurt/M : Fischer, 1992, p. 235.

3. Les chiffres entre parenthèses renvoient au livre de poche du Fischer Verlag: Franz Werfel: Jeremias. Höret die Stimme. Frankfurt/M : Fischer, 1991.

4. À ce propos, voir Paul Tillich : Le Courage d'être, Paris, Casterman, 1967, p. 54 sq.

5. Op. cit., p. 25 : «Wenn ich mich auch hundertmal selbst betrügen möchte, so glaube ich doch fest an Sinn und Folgerichtigkeit... Vielleicht kann ich auch wegen dieses Glaubens nicht mehr arbeiten. Er unterscheidet mich von den anderen. Alles, was heute geschrieben wird, beruht auf dem Gegenteil, auf der Überzeugung von der Sinnleere und Zusammenhanglosigkeit und darum auf tollen Assoziationen... Die andern sind frei dazu, ich bin nicht frei... Ganz tief in mir wohnt noch immer der alte kausale Gott, der Gott des Wenn-Dann... Er ist hier zu Hause... Wer weiß, vielleicht hat er mich hergelockt... ». Voir aussi, p. 26.

6. Ibid., p. 84 : «Priester wahren die Ordnung des Herrn, ohne abzuweichen ».

7. Ibid., p. 83 : «Sie mischen sich mit geifernder Stimme in die Geschäfte des Königs und seiner Fürsten; das Volk aber wiegeln sie mit ihren Weissagungen auf ».

8. Ibid., p. 125 : "Jetzt aber blickte et fremd und gedankenlos auf diesen Leichnam nieder, obgleich er wußte, daß Hilkijahs Tode mit seiner Künderrede im Tempel in einem verborgenen Zusammenhang stand. »

9. Ibid., p. 306: «Versöhnung gibt es hier nicht, nur Jirmijahs Tod kann das Herz der Brüder erleichtern. [...] Jirmijah ist des Glaubens, daß die Brüder ihn erbarmungslos der Gewalt des Königs ausliefern werden, wenn anders sie selbst sich dazu nicht entschließen, ihn mit eigenen Händen aus ihrem Wege zu räumen. »

10. Ibid., p. 320 : «Wiederum erfüllte sich an ihm das geheimnisvolle Gesetz der Aussonderung, das die Heimat zur eigentlichen Fremde machte und die Nächsten zu den Fernsten ».

11. Ibid., p. 297 : «Jojakim wurde vom Geiste der Vermischung umgetrieben, während der Herr doch vom Hause David den Geist der Entmischung und Sonderung forderte » et p. 330.

12. Ibid., p. 526 : «Ein Grundstein und ein Eckstein hättest du werden können.. Nun aber bist du nur ein toter Block, der im Wege liegt... »

13. Ibid., p. 428 : « Nicht Tempel, nicht Opfer und Opfergebet sind wichtig dem Herrn!»

14. Ibid., p. 403 : " auch die Schau der Sterne ist kein echtes Gesicht, ebensowenig wie die Schau der Toten ein echtes Gesicht war. [...] Die Sterne sind eitel. [...] Beinahe lasterhaft erscheint ihm jetzt ihre eisige Einsamkeit, verrucht die abgestimmte Harmonie der In-sich-selbst-Versunkenen und Von-sich-selbst-Erfüllten. [...] Wie die Kâ's in der Amenti nur Masken sind, die das schreiende Nichts verbergen, so sind auch die Sterne in Babels Schau nur Masken mit all ihren Strahlen und Kräften ».

15. Ibid., p. 406 : «Hört, Mardukh und alle Gestirne, der Ewige ist unser Gott, der Ewige ist einzig!».

16. Cf. André Ridouard : Jérémie l'épreuve de la foi, Paris, Cerf, 1983, p. 100.

17. Op. cit., p. 98 : « Die Seherin aber wurde von ihrer Schau hochgerissen, [...] Und jetzt brach aus der gebrechlichen ein gewaltig lauter Jammer, der sich zu einem gellenden Schrei steigerte ».

18. Ibid., p. 287 : «Jirmijah wurde zu Boden geworfen, ins Gesicht geschlagen, getreten », et p. 475.

19. Ibid., p. 488-489: « Meine Helden, tuet nach eurem Belieben mit ihm... ».

20. Ibid., p. 489 : « Sie schlugen ihn, sie spien ihm ins Gesicht, sie verunreinigten seine Kleider». 
21. Voir aussi Hans Wagener: Understanding Franz Werfel. University of South Carolina Press, 1993, pp. 126-127.

22. Cf. Karl Jaspers : Die maßgebenden Menschen. München : Piper, 2000, «Zur Wesensidee Jesu gehört das Leiden, das schrecklichste, uneingeschränkte, grenzenlose Leiden, das im grausamsten Tod vollendet wird. [...] Diese Leidensfähigkeit und Leidenswahrhaftigkeit ist geschichtlich einzig. [...] Jesus ist ein Gipfel dieses Leidenkönnens », p. 114.

23. Op. cit., p. 178 : «Jirmijah hält den Kopf des Verwundeten im Schoß. »

24. Ibid., p. 381 : « Und Jirmijah erkannte, daß ihn Konjah unversöhnlich häßte. Da erniedrigte er sich und diente fortan dem Unglücklichen nur als Knecht, der ihm das Essen zutrug, aufwartete und für die Notdurft des Leibes sorgte. »

25. Ibid., p. 525 : « Er schleppte sich zu dem Gerichteten aufs Gerüst und bettete den Kopf mit den blutig verkohlten Höhlen in den Schoß. »

26. Ibid., p. 357 : «Ihn ergriff Erbarmen mit den Männern und Weibern ringsum, die totengleich dreinblickten, schwer atmeten und manchmal in dumpfe Rufe der Verzweiflung ausbrachen. »

27. Ibid., p. 108 : «Er wollte nicht begreifen, daß der Gott Israels kein Gott ausschließlich für Israel, sondern ein Gott der ganzen Welt durch Israel ist».

28. Ibid., p. 120 : «Eines aber tat not. Nicht er durfte erregt nach Worten suchen, nicht er um den Ausdruck der eigenen Gedanken ringen. Seine Aufgabe war es, dem Herrn wie im Schlafe zu vertrauen, seinen eigenen Lebens- und Denkgeist auszuscheiden und seine Sinne fast bis an die Todesgrenze von sich selbst zu entleeren. »

29. Ibid., p. 468 : «Nun hatte Adonaï aus seinem Antlitz das Zeichen Hilkijahs, die sichtbare Urkunde der Väter und Vorväter für immer gelöscht. [...] Der Herr hatte ihm ein neues Antlitz gegeben, damit er sich selbst und seinem ererbten Anteil nicht mehr ähnlich sehe. [...] Doch ihm erschien sein neues Antlitz, diese äußere Wiedergeburt innerhalb eines einzigen Lebens, als geheimnisvolle Ehre.».

30. Ibid., p. 491 : « Mit einem Schlage verstand Jirmijah das Grauen und den Ekel, die den Herrn erfüllen mußten, wenn er sein Wort herabsandte, daß es aus einem menschlich-leiblichen Gehäuse ertöne. Jirmijahs eigene schlimme Lage in diesem Kotgrabe entsprach der schlimmen Lage des göttlichen Wortes, das unermüdlich immer wieder niederstieg, um Israel zu retten, und immer wieder ungehört verschmachten muß. »

31. Ibid., p. 541 : « Längst hatte ihm sein Meister eröffnet, daß er, Jirmijah, an den Rest gebunden sei, an die Hefe und Neige, die nun bald in das Haus der Knechtschaft einzog, um dort zu versinken für immer. »

32. Ibid., p. 551 : « Jirmijah erschrickt. [...] « Damit du lebest !» [...] Das heißt : Damit du den Tod überwindest, habe ich solches an dir getan. Damit Israel das Gericht überwinde, habe ich es gehalten. Aus meiner Hand strömt nur Leben, wie könntest du, der meiner Hand entströmt ist, sterben und vergeblich gewesen sein!?»

33. Ibid., p. 178 : «Und er gedachte von Stunde zu Stunde mehr, dem Herrn zu kündigen und sich der Aussonderung zu entziehen. Denn diese war ja auch nur ein Bund, den man brechen konnte, wie Adonaï den Bund mit Josijah gebrochen hatte. »

34. Ibid., p. 237-238 : «Jirmijah hatte den Pfeil wider den Herrn von seiner Seele abgeschnellt. [...] Durch das, was er jetzt sann, tat und vorhatte, verfluchte er sich selbst, nur um den Herrn zu strafen, der jeden tötete, den er liebte, und ihn entblößte und verdarb wie seinen ärgsten Feind. "

35. Ibid., p. 280 : « Da bin ich vor dir geflohen und hab mich versteckt... Aber du hast mich nicht losgelassen, du warst in meinem Herzen verschlossen und in meinen Gebeinen, du brennendes Feuer... Du bist in mir, du brennendes Feuer, ich ertrage es nicht und muß vergehn...»

36. Ibid., p. 343 : « Nach dem Tode der Mutter hatte er keine Haderkraft wider den Herrn mehr, der endgültig der Stärkere geblieben war. » 
37. Ibid., p. 289 : « Ein großes Verzagtsein zog in sein Herz ein. Und aus dem Verzagtsein wurde kleinmütige Schwäche und aus der kleinmütigen Schwäche wurde atemenge Menschenfurcht und aus der atemengen Menschenfurcht zähneklappernde Todesangst. Seine Hände klammerten sich an die Säule. Sein Kopf schwankte hin und her. Er war verloren. »

38. Ibid., p. 377 : «Dennoch hätte Jirmijah die Entscheidung zwischen Bleiben und Gehen nicht aus sich selbst getroffen, wäre nicht eine sonderbare Raunung ergangen, neu und überraschend wie selten noch.»

39. Ibid., p. 102 : «Einer, den kein Name nennt, hat Himmel und Erde, Tag und Nacht, Meer und Land, Tier und Mensch geschaffen. Er war immer und ist und wird immer sein. »

40. Ibid., p. 326 : «Der Anblick der Fronsklaven auf den Äckern, die auch die seinen waren, durchzuckte Jirmijah mit Schreck und Scham. Er, der von den Königen kühn die Erfüllung der Lehre forderte, hatte mit feige geschlossenen Augen auf seinem eigenen Grunde die NichtErfüllung des hohen Gebotes geduldet. Wenn der Tag der Verrechnung kam, stand er nicht reiner da als Obadjah und Joel und mußte sich als ein Mitschuldiger selbst verdammen.»

41. Ibid., p. 311 : «Solchen gnädigen Stunden der herablassenden Mitarbeit Adonais standen aber wie immer Stunden äußerster Verdammnis gegenüber, wo sich ihm nicht nur das Wort entzog, sondern die innere Leere mit dem Ekel der Amenti überwältigte.»

42. Voir aussi p. 294 : «Das Verzagtsein, die Schwäche, die Menschenfurcht, die Todesangst war bis auf den letzten Schatten aus seiner Seele gewichen».

43. Ibid., p. 100-101: «Eine schadhafte Stelle seines Wesens blieb dunklen Einflüsterungen offen, denen er erlag. Ihm genügte es nicht, zu sein, was er war, das Geschöpf. Mit dem Übergriff dieses Hochmutes zerstörte er die Ordnung des Herrn und sein eigenes Leben, das fortan die Herrschaft über die Erde verlor und dem Tod anheimfiel ».

44. Ibid., p. 554 : «Er ahnt: Durch dieses Neue ist die Krankheit überwunden, die Unordnung in ihm zur Ordnung gekommen auf einem unbekannten Heilungsweg. »

45. Ibid., p. 555 : « sein eigenes Selbst ist fest und mutig geworden wie noch nie... ».

46. Ibid., p.556: «Geistige Freude dehnt seine Brust, denn er weiß, daß die Stimme ungeschwächt auch in dieser Zeit und Welt spricht ».

\section{RÉSUMÉS}

L'étude du roman de Franz Werfel Jeremias. Höret die Stimme (1937) porte sur le travail de réécriture entrepris par l'auteur à partir d'abondantes sources bibliques, exégétiques et historiques. Elle vise à dégager la fonction de ce récit pour Werfel lui-même et pour son époque. L'étude montre que le romancier reprend et amplifie trois aspects essentiels du livre de Jérémie : la protestation contre le monde, la souffrance endurée en retour et l'affirmation du courage de la confiance qui s'appuie sur une expérience unique et personnelle de la transcendance. Werfel donne de Jérémie une image à la fois conforme à la figure vétérotestamentaire du prophète biblique et proche de la figure néotestamentaire du Christ, assumant ainsi le double héritage de la tradition judéo-chrétienne. La réécriture de l'histoire de Jérémie permet d'une part à Werfel lui-même de surmonter une grave crise existentielle et de retrouver ainsi sa force créatrice, et d'autre part d'adresser à son époque un appel indirect à la résistance au nazisme et une mise en garde contre les conséquences désastreuses de cette idéologie perverse. L'histoire, hélas, montrera que Werfel, pas plus que Jirmijah, n'aura réussi à inverser le cours des événements. 
Die Untersuchung befaßt sich mit der Frage, wie Franz Werfel für seinen Roman Jeremias. Höret die Stimme die biblischen, exegetischen und historischen Quellen verarbeitet. Ziel der Analyse ist herauszuarbeiten, welche Funktion der Roman für Werfel selbst und für seine Epoche erfüllt. Der Romancier übernimmt drei wesentliche Aspekte aus dem Buche Jeremias und entwickelt sie weiter : die Auflehnung gegen die bestehende Ordnung, das dafür ausgestandene Leid und die Behauptung des Mutes zum Vertrauen, der auf einer einzigartigen und persönlichen Erfahrung der Transzendenz basiert. Das von Werfel entworfene Bild des Jeremias weist zugleich Züge des alttestamentlichen Propheten und des neutestamentlichen Christus auf, das zeigt also, daß das doppelte Erbe der jüdisch-christlichen Tradition im Roman Aufnahme findet. Die Verarbeitung der Geschichte Jeremias erfüllt einen doppelten Zweck: sie hilft Werfel, eine schwere existentielle Krise zu überwinden und so seine künstlerische Schaffenskraft neu zu beleben und ermöglicht es ihm, eine indirekte Aufforderung zum Widerstand gegen den Nationalsozialismus auszusprechen und eine Warnung vor den unheilvollen Folgen dieser pervertierten Ideologie an seine Zeit zu richten. Die Geschichte wird zeigen, daß Werfel, wie Jirmijah, in seiner Mahnerrolle gescheitert ist.

\section{AUTEUR}

\section{BERNARD BACH}

Université Charles-de-Gaulle - Lille 3 